\title{
Laplace Decomposition Method for Time-Fractional Fornberg-Whitham Type Equations
}

\author{
Thilagarajah Mathanaranjan, Dayalini Vijayakumar \\ Department of Mathematics and Statistics, University of Jaffna, Jaffna, Northern Province, Sri Lanka \\ Email: mathanaranjan@gmail.com, yali.kumar1994@gmail.com
}

How to cite this paper: Mathanaranjan, T. and Vijayakumar, D. (2021) Laplace Decomposition Method for Time-Fractional Fornberg-Whitham Type Equations. Journal of Applied Mathematics and Physics, 9, 260-271.

https://doi.org/10.4236/jamp.2021.92019

Received: August 25, 2020

Accepted: February 22, 2021

Published: February 25, 2021

Copyright $\odot 2021$ by author(s) and Scientific Research Publishing Inc. This work is licensed under the Creative Commons Attribution International License (CC BY 4.0).

http://creativecommons.org/licenses/by/4.0/

\begin{abstract}
In this study, the closed form of series solutions of the original and modified nonlinear time-fractional Fornberg-Whitham equations are derived by means of the Laplace decomposition method (LDM). The fractional order derivatives are expressed in the sense of Caputo. For the specific choice of parameters, the obtained solutions are compared with the exact solutions to validate the accuracy of this method. Numerical solutions are represented graphically which illustrate the behavior of the solutions. Further, the computations express that the above method is straightforward, and it desires the smaller size of computation.
\end{abstract}

\section{Keywords}

Fractional Derivative, Fractional Fornberg-Whitham Equation, Laplace Transformation, Adomian Decomposition Method

\section{Introduction}

The fractional order nonlinear differential equations (NDE) have been the focus of many studies due to their numerous applications in the areas of mathematical physics and engineering [1]. In recent years, it has been a great interest to find the analytical methods for solving fractional NDE. Exact solutions of most of the fractional NDE cannot be found easily, consequently, analytical and approximate methods have been used. Some of the recent approximate methods for solving NDE include the Variational iteration method (VIM) [2], Differential transformation method (DTM) [3], Homotopy-perturbation method (HPM) [4], Adomian decomposition method (ADM) [5] [6] [7] and Homotopy analysis method (HAM) [8]. 
Nonlinear partial differential equations (PDE) widely express complex mechanical and physical phenomenon. Many nonlinear PDE, such as the Korteweg de Vries equation, Camassa Holm equation and Degasperis-Procesi equation have different types of traveling wave solutions. The Fornberg-Whitham equation is one such type of equation which was first introduced for illustrating the qualitative behaviour of wave breaking [9]. Recently, various types of methods are successfully applied to find extract travelling wave solutions and numerical solutions to the nonlinear Fornberg-Whitham equation [10] [11] [12].

The original Fornberg-Whitham equation with time-fractional of the form [13]

$$
u_{t}^{\alpha}-u_{x x t}+u_{x}=u u_{x x x}-u u_{x}+3 u_{x} u_{x x}
$$

with the initial condition

$$
u(x, 0)=\mathrm{e}^{x / 2}, x>0,
$$

where $u(x, t)$ is the fluid velocity. For $\alpha=1$ the Equation (1) becomes a classical Fornberg-Whitham equation. This equation was utilized to study the qualitative behaviours of wave-breaking and obtained a peaked limiting travelling wave solution of the form

$$
u(x, t)=A \mathrm{e}^{-1 / 2(\mid x-4 / 3 t)},
$$

where $A$ is constant [14].

The modified time-fractional Fornberg-Whitham equation is considered by changing the nonlinear term $u u_{x}$ in Equation (1) to $u^{2} u_{x}$ as follows [15]:

$$
u_{t}^{\alpha}-u_{x x t}+u_{x}=u u_{x x x}-u^{2} u_{x}+3 u_{x} u_{x x},
$$

with the initial condition

$$
u(x, 0)=a \operatorname{sech}^{2}(b x) .
$$

For $\alpha=1$, using the bifurcation theory method which is widely used to solve the nonlinear differential equations, the explicit peakon and soliton solutions are obtained by the form

$$
u(x, t)=a \operatorname{sech}^{2}(b(x+4 a / 3 t))
$$

where $a=-3 / 4(5-\sqrt{15})$ and $b=1 / 20 \sqrt{10(5-\sqrt{15})}$ [16]. Further, numerical solutions time fractional Fornberg-Whitham type equations are obtained by using various methods [17] [18] [19] [20].

In the present study, we consider the original and modified time-fractional Fornberg-Whitham equation to find the series solutions through Laplace decompasition method (LDM). The LDM is combined with the Adomian decomposition method and the Laplace transform. Our manuscript is structured as follows: In Section II, we list some basic definitions and properties of the fractional calculus theory. A brief summary of Laplace decomposition method is given in Section III and the implementation of LDM is given in Section IV. Then the following two subsections, the series solutions of the time-fractional Forn- 
berg-Whitham equation and modified Fornberg-Whitham equation are provided. Finally, the concluding remark to the proposed results is given in Section $\mathrm{V}$.

\section{Preliminaries}

We provided some definitions and basic properties of fractional calculus theory and then we give the basics of Laplace transformation which will be applied later in this work.

Definition 2.1 Let a function $f \in C_{\mu}, \mu \geq-1$ and $\alpha \geq 0$, then the Riemann-Liouville fractional integral of order $\alpha$ is defined by

$$
J^{\alpha} f(x)=\frac{1}{\Gamma(\alpha)} \int_{0}^{x}(x-t)^{\alpha-1} f(t) \mathrm{d} t, \quad x>0,
$$

Some basic properties of the operator $J^{\alpha}$ are as following [21]:

1) $J^{\alpha}\left(J^{\beta} f(x)\right)=J^{\beta}\left(J^{\alpha} f(x)\right)$,

2) $J^{\alpha}\left(J^{\beta} f(x)\right)=J^{\alpha+\beta} f(x)$,

3) $J^{\alpha} x^{\gamma}=\frac{\Gamma(\gamma+1)}{\Gamma(\alpha+\gamma+1)} x^{\alpha+\gamma}$,

where $\alpha, \beta \geq 0$ and $\gamma>-1$.

Caputo and Mainardi [22] modified the fractional differentiation operator which overcomes the discrepancy of Riemann-Liouville derivative.

Definition 2.2 The Caputo fractional derivative of order $\alpha>0$ is defined by

$$
D^{\alpha} f(x)=J^{m-\alpha} D^{m} f(x)=\frac{1}{\Gamma(m-\alpha)} \int_{0}^{x}(x-t)^{m-\alpha-1} f^{(m)}(t) \mathrm{d} t,
$$

where $m-1<\alpha \leq m, m \in N, x>0, f \in C_{-1}^{m}$.

Also the derivative satisfies the following properties:

1) $D^{\alpha}\left(J^{\alpha} f(x)\right)=f(x)$

2) $J^{\alpha}\left(D^{\alpha} f(x)\right)=f(x)-\sum_{k=0}^{m-1} f^{(k)}\left(0^{+}\right) \frac{x^{k}}{k !}, x>0$

Details study of fractional derivatives and integrals can be found in [21] [22] [23].

Definition 2.3 The Laplace transform of a given function $f(t)$ is defined by

$$
F(s)=\mathcal{L}\{f(t)\}=\int_{0}^{\infty} f(t) \mathrm{e}^{-s t} \mathrm{~d} t,
$$

whenever the improper integral converges.

Some properties of the Laplace transform are given below:

1) $\mathcal{L}\left\{f^{(n)}(t)\right\}=s^{n} \mathcal{L}\{f(t)\}-\sum_{k=0}^{n-1} s^{n-1-k} f^{(k)}(0)$,

2) $\mathcal{L}\left\{t^{n} f(t)\right\}=(-1)^{n} F^{(n)}(s)$.

\section{The Laplace Decomposition Method}

In order to explain the central idea of this method, first we consider a general fractional nonlinear PDE with the initial condition of the following form: 


$$
\begin{gathered}
D_{t}^{\alpha} u(x, t)+R u(x, t)+N u(x, t)=g(x, t) \\
u(x, 0)=f(x)
\end{gathered}
$$

where $D_{t}^{\alpha} u(x, t)$ denotes the Caputo fractional derivative of $u(x, t), R$ is a linear operator, $N$ is a nonlinear operator and $g$ is a non-homogeneous term.

We take the Laplace transform on both sides of Equation (8) and then using the differentiation property of Laplace transform, we get,

$$
\mathcal{L}[u(x, t)]=\frac{f(x)}{s}+\frac{1}{s^{\alpha}} \mathcal{L}[g(x, t)]-\frac{1}{s^{\alpha}} \mathcal{L}[R u(x, t)+N u(x, t)] .
$$

Now, taking the inverse Laplace transform to Equation (9)

$$
u(x, t)=G(x, t)-\mathcal{L}^{-1}\left[\frac{1}{s^{\alpha}} \mathcal{L}[R u(x, t)+N u(x, t)]\right] .
$$

where $G(x, t)$ is source term and give initial condition.

The series solution of the Adomian decomposition method is given by,

$$
u(x, t)=\sum_{n=0}^{\infty} u_{n}(x, t),
$$

with the nonlinear term $N u(x, t)$ can be decomposed as

$$
N u(x, t)=\sum_{n=0}^{\infty} A_{n}\left(u_{0}, u_{1}, \cdots, u_{n}\right),
$$

Adomian polynomials $A_{n}$ can be derived by particular algorithms constructed by Adomian [24] or alternatively by Wazwaz [25]. The formula for $A_{n}$ is given by

$$
A_{n}=\frac{1}{n !} \frac{\mathrm{d}^{n}}{\mathrm{~d} \lambda^{n}}\left[g\left(\sum_{k=0}^{n} \lambda^{k} u_{k}\right)\right]_{\lambda=0} .
$$

Substituting (11) and (12) into Equation (10), we get,

$$
\sum_{n=0}^{\infty} u_{n}(x, t)=G(x, t)-\mathcal{L}^{-1}\left[\frac{1}{s^{\alpha}} \mathcal{L}\left[R \sum_{n=0}^{\infty} u_{n}(x, t)+\sum_{n=0}^{\infty} A_{n}\left(u_{0}, u_{1}, \cdots, u_{n}\right)\right]\right],
$$

From the equation (14) we get,

$$
\left\{\begin{array}{l}
u_{0}(x, t)=G(x, t), \\
u_{n+1}(x, t)=-\mathcal{L}^{-1}\left[\frac{1}{s^{\alpha}} \mathcal{L}\left[R u_{n}(x, t)+A_{n}\left(u_{0}, u_{1}, \cdots, u_{n}\right)\right]\right], n=0,1,2, \cdots
\end{array}\right.
$$

From (15), the series solution of (8) can be found

$$
u(x, t) \approx \sum_{n=0}^{k} u_{n}(x, t), \text { where } \lim _{k \rightarrow \infty} \sum_{n=0}^{k} u_{n}(x, t)=u(x, t) .
$$

In generally, the above series is convergence rapidly [26] [27] [28] [29].

\section{Implementation of LDM}

First, consider the generalized Fornberg-Whitham equation with time-fractional of the form 


$$
\begin{gathered}
u_{t}^{\alpha}-u_{x x t}+u_{x}=u u_{x x x}-u^{n} u_{x}+3 u_{x} u_{x x}, t>0,0<\alpha, \\
u(x, 0)=u_{0}(x),
\end{gathered}
$$

where $n=1$ and 2. Comparing (17) with Equation (8) we get $g(x, t)=0$ and linear term $R$ turn out to be

$$
R u=-u_{x x t}+u_{x},
$$

whereas the nonlinear term

$$
N u=u^{n} u_{x}-u u_{x x x}-3 u_{x} u_{x x}
$$

Using Equation (17), we get

$$
\left\{\begin{array}{l}
u_{0}(x, t)=u_{0}(x), \\
u_{n+1}(x, t)=-\mathcal{L}^{-1}\left[\frac{1}{s^{\alpha}} \mathcal{L}\left[-u_{n x x t}(x, t)+u_{n x}(x, t)+A_{n}\right]\right], n=0,1,2, \cdots
\end{array}\right.
$$

Also

$$
N_{1} u=u^{n} u_{x}-u u_{x x x}-3 u_{x} u_{x x}=\sum_{n=0}^{\infty} A_{n}
$$

Adomian polynomials $\left\{A_{n}\right\}_{n=0}^{\infty}$ can be calculated using the procedure stated in [24] as follows:

$$
\begin{gathered}
A_{0}=u_{0}^{n} u_{0 x}-u_{0} u_{0 x x x}-3 u_{0 x} u_{0 x x}, \\
A_{1}=n u_{0}^{n+1} u_{1} u_{0 x}+u_{0}^{n} u_{1 x}-u_{1} u_{0 x x x}-u_{0} u_{1 x x x}-3 u_{1 x} u_{0 x x}-3 u_{0 x} u_{1 x x}, \\
A_{2}=\frac{1}{2} n^{2} u_{0}^{n-2} u_{1}^{2} u_{0 x}-\frac{1}{2} n u_{0}^{n-2} u_{1}^{2} u_{0 x}+n u_{0}^{n-1} u_{2} u_{0 x}+n u_{0}^{n-1} u_{1} u_{1 x}+u_{0}^{n} u_{2 x} \\
-u_{2} u_{0 x x x}-u_{1} u_{1 x x x}-u_{0} u_{2 x x x}-3 u_{2 x} u_{0 x x}-3 u_{1 x} u_{1 x x}-3 u_{0 x} u_{2 x x}, \\
A_{3}=\frac{1}{6} n^{3} u_{0}^{n-3} u_{1}^{3} u_{0 x}+\frac{1}{3} n u_{0}^{n-3} u_{1}^{3} u_{0 x}-\frac{1}{2} n^{2} u_{0}^{n-3} u_{1}^{3} u_{0 x}-\frac{1}{2} n u_{0}^{n-2} u_{1}^{2} u_{1 x} \\
+\frac{1}{2} n^{2} u_{0}^{n-2} u_{1}^{2} u_{1 x}-n u_{0}^{n-2} u_{1} u_{2} u_{0 x}+n^{2} u_{0}^{n-2} u_{1} u_{2} u_{0 x}+n u_{0}^{n-1} u_{3} u_{0 x} \\
+n u_{0}^{n-1} u_{2} u_{1 x}+n u_{0}^{n-1} u_{1} u_{2 x}+u_{0}^{n} u_{3 x}-u_{3} u_{0 x x x}-u_{2} u_{1 x x x}-u_{1} u_{2 x x x} \\
-u_{0} u_{3 x x x}-3 u_{3 x} u_{0 x x}-3 u_{2 x} u_{1 x x}-3 u_{1 x} u_{2 x x}-3 u_{0 x} u_{3 x x},
\end{gathered}
$$

and so on. Using the above recursive relationships with Adomian polynomials the decomposition series (16) can be found as follows:

$$
\begin{gathered}
u_{0}(x, t)=u_{0}, \\
u_{1}(x, t)=\mathcal{L}^{-1}\left[\frac{1}{s} \mathcal{L}\left\{u_{0 x x t}-a u_{0 x}+A_{0}\right\}\right], \\
u_{2}(x, t)=\mathcal{L}^{-1}\left[\frac{1}{s} \mathcal{L}\left\{u_{1 x x t}-a u_{1 x}+A_{1}\right\}\right] \\
u_{3}(x, t)=\mathcal{L}^{-1}\left[\frac{1}{s} \mathcal{L}\left\{u_{2 x x t}-a u_{2 x}+A_{2}\right\}\right], \\
\vdots \\
u_{n}(x, t)=\mathcal{L}^{-1}\left[\frac{1}{s} \mathcal{L}\left\{u_{n-1 x x t}-a u_{n-1 x}+A_{n-1}\right\}\right],
\end{gathered}
$$


and so on. In the following two subsection, we utilize the above results for two initial value problems associated with the original and modified time-fractional Fornberg-Whitham equations in order to find the series solutions.

\subsection{Solution of the Original Time-Fractional Fornberg-Whitham Equation}

For $n=1$, the Equation (17) reduces to the original time-fractional Fornberg-Whitham equation as follows;

$$
\begin{gathered}
u_{t}^{\alpha}-u_{x x t}+u_{x}=u u_{x x x}-u u_{x}+3 u_{x} u_{x x}, t>0,0<\alpha \leq 1, \\
u(x, 0)=\mathrm{e}^{x / 2}, x>0 .
\end{gathered}
$$

For $\alpha=1$, the exact solution of peaked limiting form of the travelling wave is given by [14]

$$
u(x, t)=A \mathrm{e}^{-1 / 2(\mid x-4 / 3 t)} .
$$

By using the above recursive relationships and Adomian polynomials $A_{n}$, we find the terms of decomposition series (16) as follows:

$$
\begin{gathered}
u_{0}(x, t)=\mathrm{e}^{x / 2}, \\
u_{1}(x, t)=-\frac{\mathrm{e}^{x / 2} t^{\alpha}}{2 \Gamma[1+\alpha]}, \\
u_{2}(x, t)=+\frac{\mathrm{e}^{x / 2} t^{2 \alpha}}{4 \Gamma[1+2 \alpha]}-\frac{\mathrm{e}^{x / 2} t^{-1+2 \alpha}}{8 \Gamma[2 \alpha]}, \\
u_{3}(x, t)=-\frac{\mathrm{e}^{x / 2} t^{3 \alpha}}{8 \Gamma[1+3 \alpha]}+\frac{\mathrm{e}^{x / 2} t^{-1+3 \alpha}}{8 \Gamma[3 \alpha]}-\frac{\mathrm{e}^{x / 2} t^{-2+3 \alpha}}{32 \Gamma[-1+3 \alpha]}, \\
u_{4}(x, t)=+\frac{\mathrm{e}^{x / 2} t^{4 \alpha}}{16 \Gamma[1+4 \alpha]}-\frac{3 \mathrm{e}^{x / 2} t^{-1+4 \alpha}}{32 \Gamma[4 \alpha]}+\frac{3 \mathrm{e}^{x / 2} t^{-2+4 \alpha}}{64 \Gamma[-1+4 \alpha]}-\frac{\mathrm{e}^{x / 2} t^{-3+4 \alpha}}{128 \Gamma[-2+4 \alpha]},
\end{gathered}
$$

and so on. Consequently, the approximate analytical solution of (22) is found in the closed form of series as follows:

$$
u(x, t)=\lim _{k \rightarrow \infty}\left[\mathrm{e}^{x / 2}+\sum_{n=1}^{k} \sum_{i=0}^{n-1}\left(\begin{array}{c}
n-1 \\
i
\end{array}\right)\left(-\frac{1}{2}\right)^{n+i} \frac{\mathrm{e}^{x / 2} t^{n \alpha-i}}{\Gamma[n \alpha+1-i]}\right] .
$$

To verify the accuracy of the LDM, we compare the numerical values of approximate solutions with the exact solutions in Table 1 . These numerical values show that LDM yields a good approximate solution, even for the lowest order approximate solution. The accuracy of solution can be improved by taking higher-order approximate solutions.

In Figure 1, the approximate solution and the exact solution are plotted. its leads to get the behavior of the approximate solutions are the same behavior with the exact solution. Consequently, the approximate series solutions are rabidly convergence as the exact solution. The $3 \mathrm{D}$ and $2 \mathrm{D}$ plots for different values of $\alpha$ are presented in Figure 2 and Figure 3. 


\subsection{Solution of Modified Time-Fractional Fornberg-Whitham Equation}

For $n=2$, the Equation (17) reduces to the modified time-fractional Fornberg-Whitham equation as follows;

Table 1. Comparison of exact solution with 5th order approximate solution of Equation (22).

\begin{tabular}{|c|c|c|c|c|c|c|}
\hline$t$ & $x$ & $\alpha=0.2$ & $\alpha=0.7$ & $\alpha=1$ & Exact Solution & Abs. error $\left|u_{E x a c t}-u_{L D M}\right|$ \\
\hline \multirow{3}{*}{0.1} & 0.5 & 0.55330756 & 1.0548937 & 1.2014201 & 1.2012147 & 0.00020536966 \\
\hline & 1.5 & 0.91224994 & 1.7392257 & 1.9808069 & 1.9804683 & 0.00033859733 \\
\hline & 2.0 & 1.1713521 & 2.23321 & 2.5434064 & 2.5429716 & 0.00043476757 \\
\hline \multirow{4}{*}{0.5} & 0.5 & 0.78942785 & 0.83412887 & 0.91975844 & 0.92004441 & 0.00028597406 \\
\hline & 1.0 & 1.0136454 & 1.0710427 & 1.1809932 & 1.1813604 & 0.00036719796 \\
\hline & 1.5 & 1.3015465 & 1.375246 & 1.5164253 & 1.5168968 & 0.00047149152 \\
\hline & 2.0 & 1.6712188 & 1.7658508 & 1.9471286 & 1.94773404 & 0.00060540709 \\
\hline \multirow{4}{*}{0.9} & 0.5 & 0.78469782 & 0.7174914 & 0.70407427 & 0.70468809 & 0.00061381852 \\
\hline & 1.0 & 1.0075719 & 0.92127719 & 0.90404926 & 0.90483742 & 0.00078815858 \\
\hline & 1.5 & 1.293748 & 1.1829433 & 1.1608222 & 1.1618342 & 0.0010120157 \\
\hline & 2.0 & 1.6612053 & 1.5189293 & 1.4905252 & 1.4918247 & 0.0012994538 \\
\hline
\end{tabular}
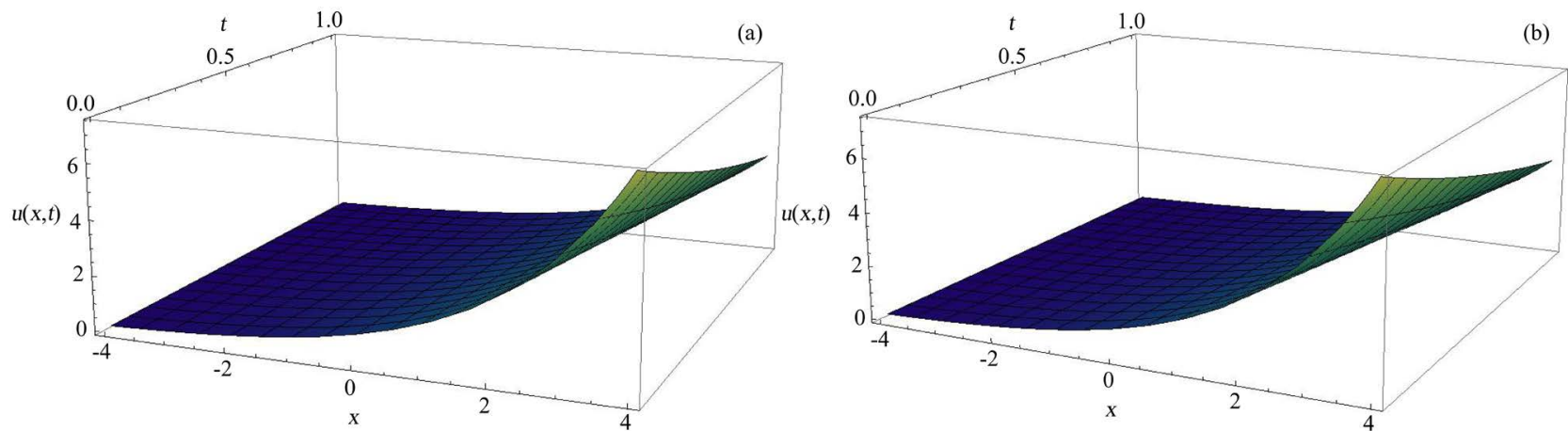

Figure 1. The exact solution is shown in the figure (a) in comparison with the 5th order approximate solution is shown in (b) when $\alpha=1$ of Equation (22).
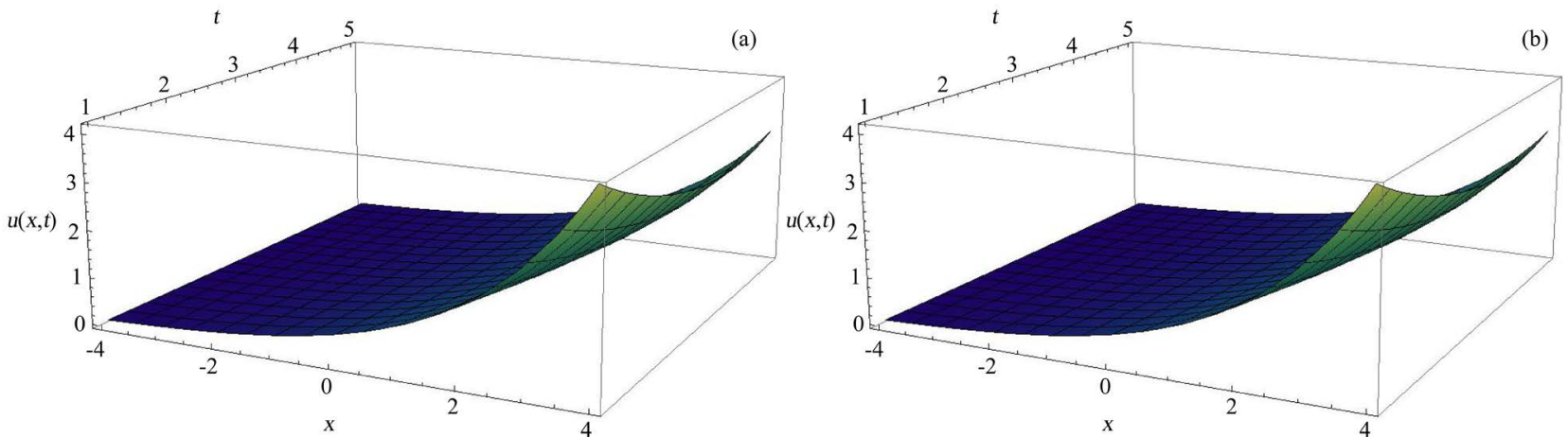

Figure 2. The behavior of the 5th order approximation solutions for $\alpha=0.2$ (a) and $\alpha=0.6$ (b) of Equation (22). 


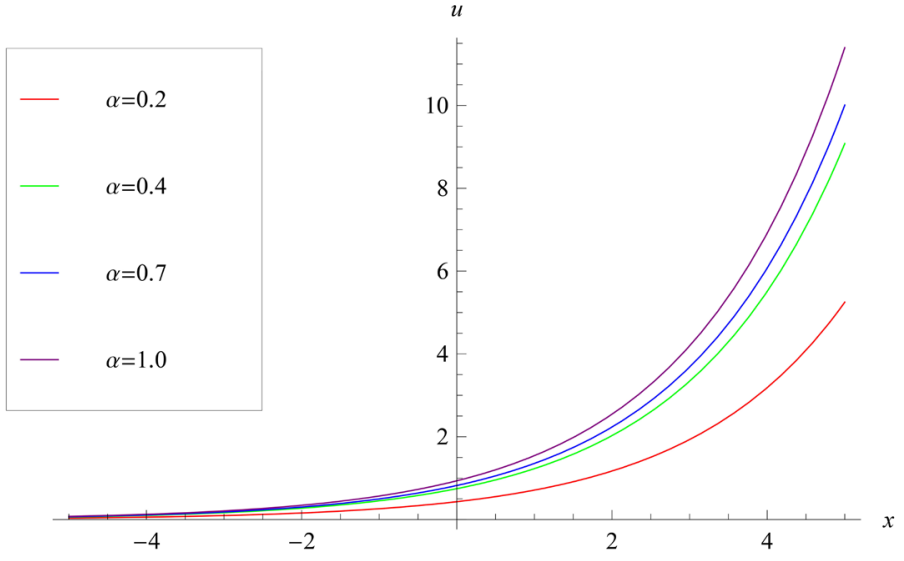

Figure 3. The 5th order of approximate solutions for different values of $\alpha$ at $t=0.1$ of Equation (22).

$$
\begin{gathered}
u_{t}^{\alpha}-u_{x x t}+u_{x}=u u_{x x x}-u^{2} u_{x}+3 u_{x} u_{x x}, t>0,0<\alpha \leq 1, \\
u(x, 0)=a \operatorname{sech}^{2}(b x),
\end{gathered}
$$

For $\alpha=1$, the explicit peakon and soliton solution of Equation (25) is given by [16]

$$
u(x, t)=a \operatorname{sech}^{2}(b(x+4 a / 3 t)) .
$$

By using the above recursive relationships and Adomian polynomials $A_{n}$, we find the terms of decomposition series (16) as follows:

$$
\begin{gathered}
u_{0}(x, t)=a \operatorname{sech}^{2}(b x), \\
u_{1}(x, t)=\frac{c_{1_{0}} a \operatorname{sech}^{2}(b x) t^{\alpha}}{\Gamma[1+\alpha]}, \\
u_{2}(x, t)=+\frac{c_{2_{0}} a \operatorname{sech}^{2}(b x) t^{2 \alpha}}{4 \Gamma[1+2 \alpha]}-\frac{c_{2_{1}} a \operatorname{sech}^{2}(b x) t^{-1+2 \alpha}}{\Gamma[2 \alpha]}, \\
u_{3}(x, t)=-\frac{c_{3_{0}} a \operatorname{sech}^{2}(b x) t^{3 \alpha}}{\Gamma[1+3 \alpha]}+\frac{c_{3_{1}} a \operatorname{sech}^{2}(b x) t^{-1+3 \alpha}}{\Gamma[3 \alpha]} \\
+\frac{c_{3_{2}} a \operatorname{sech}^{2}(b x) t^{-2+3 \alpha}}{\Gamma[-1+3 \alpha]}, \\
u_{4}(x, t)=\frac{c_{4_{0}} a \operatorname{sech}^{2}(b x) t^{4 \alpha}}{16 \Gamma[1+4 \alpha]}-\frac{c_{4_{1}} a \operatorname{sech}^{2}(b x) t^{-1+4 \alpha}}{\Gamma[4 \alpha]} \\
+\frac{c_{4_{2}} a \operatorname{sech}^{2}(b x) t^{-2+4 \alpha}}{\Gamma[-1+4 \alpha]}-\frac{c_{4_{3}} a \operatorname{sech}^{2}(b x) t^{-3+4 \alpha}}{\Gamma[-2+4 \alpha]},
\end{gathered}
$$

and so on. Consequently, as the above case, the approximate analytical solution of (25) is found in the closed form of series as follows:

$$
u(x, t)=\lim _{k \rightarrow \infty}\left[a \operatorname{sech}^{2}(b x)+\sum_{n=1}^{k} \sum_{i=0}^{n-1} c_{n_{i}} \frac{a \operatorname{sech}^{2}(b x) t^{n \alpha-i}}{\Gamma[n \alpha+1-i]}\right],
$$

where, 


$$
\begin{gathered}
c_{1_{0}}=2 b\left(1+a\left(a+22 b^{2}-8 b^{2} \cosh (2 b x)\right) \operatorname{sech}(b x)^{4}\right) \tanh (b x), \\
c_{2_{0}}=2 b^{2}\left(2+\operatorname{sech}(b x)^{2}\left(-3-128 a b^{2}+a \operatorname{sech}(b x)^{2}\left(4\left(130 b^{2}+a\left(3+864 b^{4}\right)\right)\right.\right.\right. \\
-2\left(7 a+10\left(21+32 a^{2}\right) b^{2}+13280 a b^{4} \operatorname{sech}(b x)^{2}+2 a\left(5 a^{2}+960 a b^{2}\right.\right. \\
\left.\left.\left.\left.+26172 b^{4}\right) \operatorname{sech}(b x)^{4}-11 a\left(a+30 b^{2}\right)\left(a+90 b^{2}\right) \operatorname{sech}(b x)^{6}\right)\right)\right), \\
c_{2_{1}}=b^{3}\left(-26-304 a^{2}-7968 a b^{2}+\left(-33+16 a\left(9 a+326 b^{2}\right)\right) \cosh (2 b x)\right. \\
\left.-2\left(3+128 a b^{2}\right) \cosh (4 b x)+\cosh [6 b x]\right) \operatorname{sech}(b x)^{6} \tanh (b x),
\end{gathered}
$$

and so on. In Table 2, we compare the approximate solution with the exact solution of Equation (25) for some different values of $\alpha$. These numerical values show that the LDM provides more accurate approximate solutions.

Similarly as in the previous case, the approximate solutions are the same behavior with the exact solution. This is illustrated by Figure 4 . The $3 \mathrm{D}$ and $2 \mathrm{D}$ plots for some different values of $\alpha$ are presented in Figure 5 and Figure 6.

Table 2. Comparison of exact solution with 4th order approximate solution of Equation (25).

\begin{tabular}{ccccccc}
\hline$t$ & $x$ & $\alpha=0.2$ & $\alpha=0.7$ & $\alpha=1$ & Exact Solution & Abs. error $\left|u_{\text {Exact }}-u_{L D M}\right|$ \\
\hline & 0.5 & -0.84015035 & -0.84719431 & -0.84200369 & -0.84170019 & 0.00030349875 \\
0.1 & 1.0 & -0.79140244 & -0.83591408 & -0.82723018 & -0.82678633 & 0.00044385734 \\
& 1.5 & -0.74342766 & -0.81235381 & -0.8015035 & -0.8010345 & 0.00046899813 \\
& 2.0 & -0.70107965 & -0.77787419 & -0.76618668 & -0.76580255 & 0.00038413104 \\
\hline 0.5 & 0.5 & -0.81320446 & -0.84990096 & -0.84802561 & -0.84516644 & 0.0028591668 \\
& 1.0 & -0.80726139 & -0.84820141 & -0.84379648 & -0.84074121 & 0.0030552675 \\
& 1.5 & -0.7933088 & -0.83324893 & -0.82726913 & -0.82471511 & 0.0025540116 \\
& 2.0 & -0.77275166 & -0.8062717 & -0.79944392 & -0.79796266 & 0.001481259 \\
\hline 0.9 & 0.5 & -0.78865494 & -0.83899061 & -0.84577223 & -0.83899395 & 0.0067782736 \\
& 1.0 & -0.78842909 & -0.84297517 & -0.85111767 & -0.84525761 & 0.0058600603 \\
& 1.5 & -0.78148415 & -0.83403147 & -0.84330532 & -0.83966942 & 0.003635898 \\
& 2.0 & -0.76844313 & -0.81333057 & -0.82306334 & -0.82253964 & 0.00052370169 \\
\hline
\end{tabular}
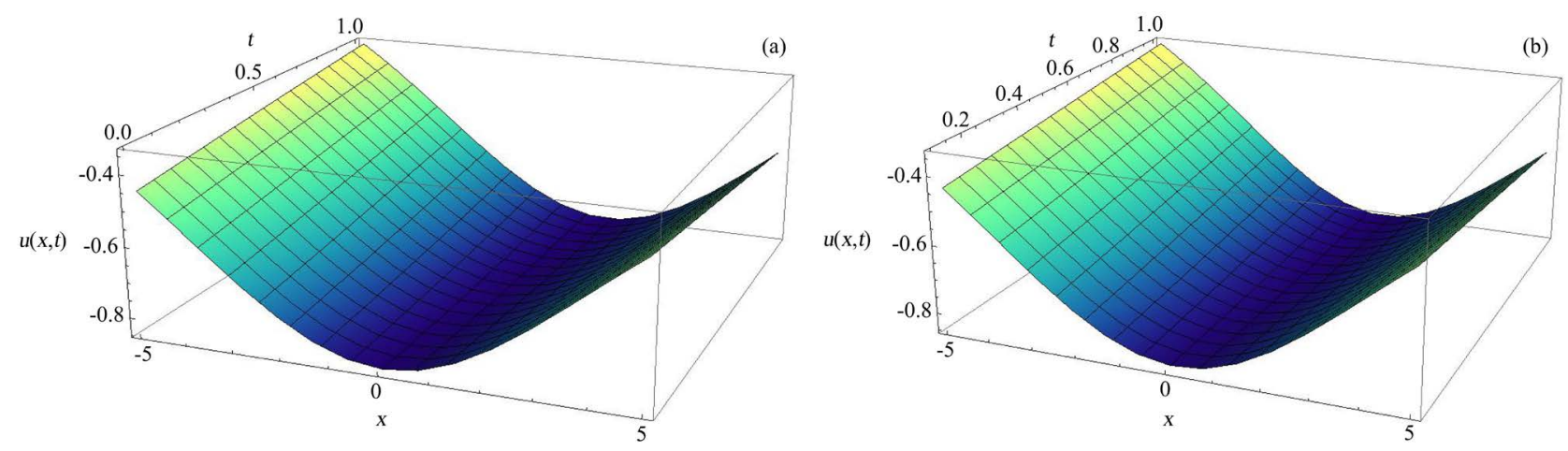

Figure 4. The exact solution is shown in the figure (a) in comparison with the 4th order approximate solution is shown in (b) when $\alpha=1$ of Equation (25). 


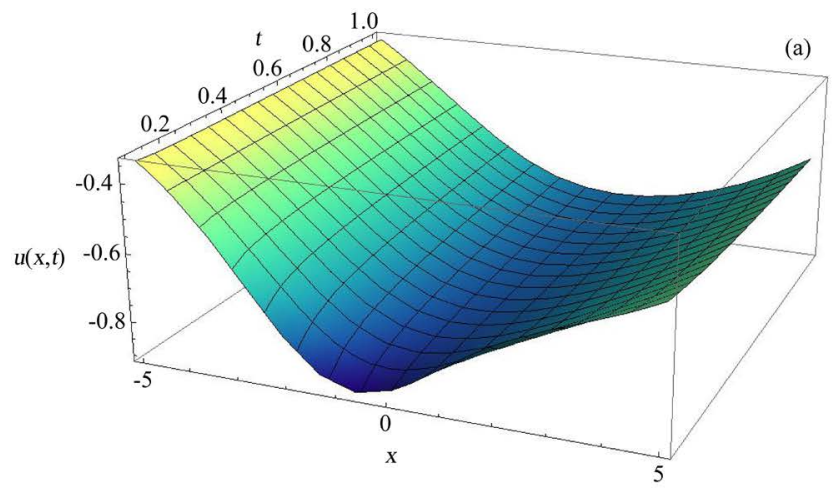

Figure 5. The behavior of the 4th order approximation solutions for $\alpha=0.2$

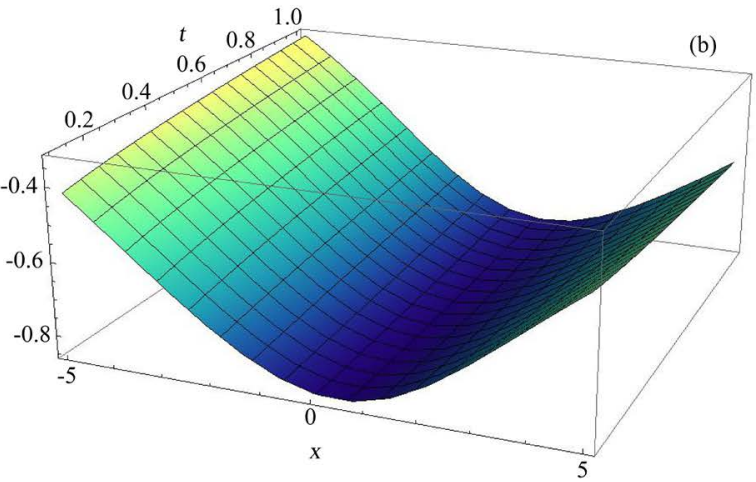

(b) of Equation (25).

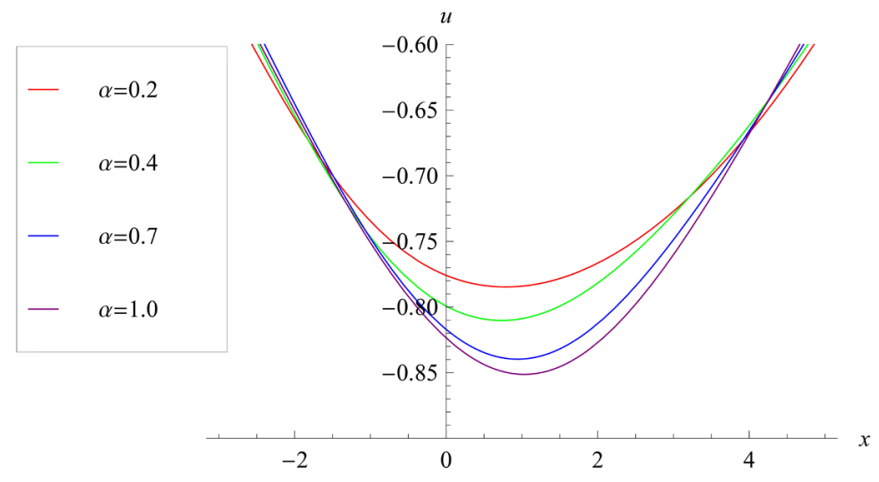

Figure 6. 4th order of approximate solutions for different values of $\alpha$ at $t=0.1$ of Equation (25).

\section{Conclusion}

In this study, the Laplace-Adomian decomposition method has been successfully applied to find a closed form of series solution of the original and modified time-fractional Fornberg-Whitham equations. For some specific choice of parameters, the approximate solutions are compared with the exact solutions. Further, the behaviours of the solutions are presented by 3D and 2D graphs. The graphs showed that the behaviors of the approximate solutions are the same behaviors of the exact solutions. As a result, we realize that the approximate solutions are rapidly convergent series as the exact solutions. Further, the computations show that the described method is easy to apply and it needs the smaller size of computation than the existing methods. Therefore, it is obvious that the LDM is very powerful, efficient and accurate for solving the nonlinear fractional differential equations.

\section{Conflicts of Interest}

The authors declare no conflicts of interest regarding the publication of this paper.

\section{References}

[1] Debnath, L. (2003) Recent Applications of Fractional Calculus to Science and Engi- 
neering. International Journal of Mathematics and Mathematical Sciences, 2003, 3413-3442. https://doi.org/10.1155/S0161171203301486

[2] Wazwaz, A.M. (2008) A Study on Linear and Nonlinear Schrödinger Equations by the Variational Iteration Method. Chaos, Solitons and Fractals, 37, 1136-1142. https://doi.org/10.1016/j.chaos.2006.10.009

[3] Ravi Kanth, A.S.V. and Aruna, K. (2009) Two-Dimensional Differential Transform Method for Solving Linear and Non-Linear Schrödinger Equations. Chaos, Solitons and Fractals, 41, 2277-2281. https://doi.org/10.1016/j.chaos.2008.08.037

[4] Sadighi, A. and Ganji, D.D. (2008) Analytic Treatment of Linear and Nonlinear Schrodinger Equations: A Study with Homotopy-Perturbation and Adomian Decomposition Methods. Physics Letters A, 372, 465-469. https://doi.org/10.1016/j.physleta.2007.07.065

[5] Khuri, S. (1998) A New Approach to the Cubic Schrodinger Equation: An Application of the Decomposition Technique. Applied Mathematics and Computation, 97, 251-254. https://doi.org/10.1016/S0096-3003(97)10147-3

[6] Rida, S.Z., El-Sherbiny, H.M. and Arafa, A.A.M. (1998) On the Solution of the Fractional Nonlinear Schrodinger Equation. Physics Letters A, 372, 553-558.

https://doi.org/10.1016/j.physleta.2007.06.071

[7] Mathanaranjan, T. and Himalini, K. (2019) Analytical Solutions of the Time-Fractional Nonlinear Schrodinger Equation with Zero and Non Zero Trapping Potential through the Sumudu Decomposition Method. Journal of Science of the University of Kelaniya Sri Lanka, 12, 21-33. https://doi.org/10.4038/josuk.v12i0.8015

[8] Khan, N.A., Jamil, M. and Ara, A. (2012) Approximate Solutions to Time Fractional Schrodinger Equation via Homotopy Analysis Method. ISRN Mathematical Physics, 2012, Article ID: 197068. https://doi.org/10.5402/2012/197068

[9] Whitham, G.B. (1967) Variational Methods and Applications to Water Wave. Proceedings of the Royal Society of London. Series A, 299, 6-25.

https://doi.org/10.1098/rspa.1967.0119

[10] Abidi, F. and Omrani, K. (2010) The Homotopy Analysis Method for Solving the Fornberg-Whitham Equation and Comparison with Adomians Decomposition Method. Computers \& Mathematics with Applications, 59, 2743-2750. https://doi.org/10.1016/j.camwa.2010.01.042

[11] Jiang, B. and Bi, Q. (2010) Smooth and Non-Smooth Traveling Wave Solutions of the Fornberg-Whitham Equation with Linear Dispersion Term. Applied Mathematics and Computation, 216, 2155-2162. https://doi.org/10.1016/j.amc.2010.03.050

[12] Lu, J. (2011) An Analytical Approach to the Fornberg-Whitham Type Equations by Using the Variational Iteration Method. Computers \& Mathematics with Applications, 61, 2010-2013. https://doi.org/10.1016/j.camwa.2010.08.052

[13] Sakar, M.G. and Erdogan, F. (2013) The Homotopy Analysis Method for Solving the Time-Fractional Fornberg-Whitham Equation and Comparison with Adomian's Decomposition Method. Applied Mathematical Modelling, 37, 8876-8885. https://doi.org/10.1016/j.apm.2013.03.074

[14] Fornberg, B. and Whitham, G.B. (1978) A Numerical and Theoretical Study of Certain Nonlinear Wave Phenomena. Philosophical Transactions of the Royal Society of London. Series A, 289, 373-404. https://doi.org/10.1098/rsta.1978.0064

[15] Saha Ray, S. and Gupta, A.K. (2015) A Numerical Investigation of Time-Fractional Modified Fornberg-Whitham Equation for Analyzing the Behavior of Water Waves. Applied Mathematicsand Computation, 266, 135-148. 
https://doi.org/10.1016/j.amc.2015.05.045

[16] He, B., Meng, Q. and Li, S. (2010) Explicit Peakon and Solitary Wave Solutions for the Modified Fornberg-Whitham Equation. Applied Mathematics and Computation, 217, 1976-1982. https://doi.org/10.1016/j.amc.2010.06.055

[17] Sakar, M.G., Erdogan, F. and Yildirim, A. (2012) Variational Iteration Method for the Time-Fractional Fornberg-Whitham Equation. Computers \& Mathematics with Applications, 63, 1382-1388. https://doi.org/10.1016/j.camwa.2012.01.031

[18] Sakar, M.G. and Erdogan, F. (2013) The Homotopy Analysis Method for Solving the Time-Fractional Fornberg-Whitham Equation and Comparison with Adomian's Decomposition Method. Applied Mathematical Modelling, 37, 8876-8885.

https://doi.org/10.1016/j.apm.2013.03.074

[19] Sontakke, B.R. and Shaikh, A. (2016) Numerical Solutions of Time Fractional Fornberg-Whitham and Modified Fornberg-Whitham Equations Using New Iterative Method. Asian Journal of Mathematics and Computer Research, 13, 66-76.

[20] Al-luhaibi, M.S. (2017) An Analytical Treatment to Fractional Fornberg-Whitham Equation. Mathematical Sciences, 11, 1-6.

https://doi.org/10.1007/s40096-016-0198-5

[21] Samko, S.G., Kilbas, A.A. and Marichev, O.I. (1993) Fractional Integrals and Derivatives: Theory and Applications. Gordon and Breach, Yverdon.

[22] Caputo, M. and Mainardi, F. (1971) Linear Models of Dissipation in Anelastic Solids. Rivista Del Nuovo Cimento, 1, 161-198. https://doi.org/10.1007/BF02820620

[23] Miller, K.S. and Ross, B. (1993) An Introduction to the Fractional Calculus and Fractional Differential Equations. Wiley, New York.

[24] Adomian, G. (1994) Solving Frontier Problems of Physics: The Decomposition Method. Kluwer Academic Publishers, Boston. https://doi.org/10.1007/978-94-015-8289-6

[25] Wazwaz, A.M. (2000) A New Algorithm for Calculating Adomian Polynomials for Nonlinear Operators. Applied Mathematics and Computation, 111, 53-69. https://doi.org/10.1016/S0096-3003(99)00063-6

[26] Cherruault, Y. (1989) Convergence of Adomian's Method. Kybernetes, 18, 31-38. https://doi.org/10.1108/eb005812

[27] Cherruault, Y. and Adomian, G. (1993) Decomposition Methods: A New Proof of Convergence. Mathematical and Computer Modelling, 18, 103-106. https://doi.org/10.1016/0895-7177(93)90233-O

[28] Abbaoui, K. and Cherruault, Y. (1994) Convergence of Adomian's Method Applied to Differential Equations. Computers \& Mathematics with Applications, 28, 103-109. https://doi.org/10.1016/0898-1221(94)00144-8

[29] Abbaoui, K. and Cherruault, Y. (1995) New Ideas for Proving Convergence of Decomposition Methods. Computers \& Mathematics with Applications, 29, 103-108. https://doi.org/10.1016/0898-1221(95)00022-Q 